Check for updates

Cite this: RSC Adv., 2018, 8, 30354

\title{
Tuning the mechanical properties of silicene nanosheet by auxiliary cracks: a molecular dynamics study $\dagger$
}

\author{
Shahriar Muhammad Nahid, ${ }^{a}$ Shahriar Nahian, ${ }^{a}$ Mohammad Motalab, (D) *a \\ Tawfiqur Rakib, ${ }^{b}$ Satyajit Mojumder (D) ${ }^{a}$ and Md Mahbubul Islam (D) ${ }^{c}$
}

Silicene has become a topic of interest nowadays due to its potential application in various electromechanical nanodevices. In our previous work on silicene, fracture stresses of single crystal and polycrystalline silicene have been investigated. Existence of defects in the form of cracks reduces the fracture strength of silicene nanosheets to a great extent. In this study, an engineering way has been proposed for improving the fracture stress of silicene nanosheets with a pre-existing crack by incorporating auxiliary cracks symmetrically in a direction perpendicular to the main crack. We call this mechanism the "Failure shielding mechanism". An extensive molecular dynamics simulation based analysis has been performed to capture the atomic level auxiliary crack-main crack interactions. It is found that the main crack tip stress distribution is significantly changed with the presence of auxiliary cracks for loading along both armchair and zigzag directions. The effects of temperature and the crack propagation speed of silicene have also been studied. Interestingly, in the case of loading along the zigzag direction, SW defect formation is observed at the tip of main crack. This leads to a reduction of the tip stress resulting in a more prominent failure shielding in case of zigzag loading than in armchair loading. Moreover, the position and length of the cracks as well as the loading directions have significant impacts on the tip stress distribution. Finally, this study opens the possibilities of strain engineering for silicene by proposing an engineering way to tailor the fracture strength of silicene.

Received 2nd June 2018

Accepted 5th August 2018

DOI: $10.1039 / c 8 \mathrm{ra0} 4728 f$

rsc.li/rsc-advances

\section{Introduction}

Two dimensional (2D) materials are a matter of interest nowadays because of their exceptional and novel physical properties. ${ }^{1-5}$ After the rise of graphene, ${ }^{6}$ the exceptional mechanical, ${ }^{7,8}$ thermal ${ }^{9}$ and electrical properties ${ }^{10-14}$ of $2 \mathrm{D}$ materials, due to their two dimensional $\pi$ bonding network, have spurred tremendous research interest. ${ }^{6,15}$ Silicene, a monolayer of silicon ( $\mathrm{Si}$ ) atoms arranged in hexagonal honeycomb lattice, is a 2D analogue of graphene. ${ }^{\mathbf{1 6 , 1 7}}$

Previously, density functional theory (DFT) calculations have been used to investigate the stability of the $2 \mathrm{D}$ honeycomb lattice of $\mathrm{Si}$ atoms. ${ }^{\mathbf{1 8 - 2 0}}$ Besides, silicene has been synthesized on $\mathrm{Ag}, \mathrm{ZrB}_{2}$ and Ir substrates by depositing Si atoms. ${ }^{21-25}$ Likewise, silicene is also a semiconductor with zero band gap. ${ }^{26}$ However,

${ }^{a}$ Department of Mechanical Engineering, Bangladesh University of Engineering and Technology, Dhaka-1000, Bangladesh.E-mail: abdulmotalab@me.buet.ac.bd

${ }^{b}$ Department of Mechanical and Chemical Engineering, Islamic University of Technology, Board Bazar, Gazipur-1704, Bangladesh

${ }^{c}$ School of Materials Engineering, Purdue University, West Lafayette, IN-47907, USA

$\dagger$ Electronic supplementary information (ESI) available. See DOI: $10.1039 / \mathrm{c} 8 \mathrm{ra} 04728 \mathrm{f}$ $\pi$ electrons of silicene are more active than graphene. Therefore, electronic properties of silicene can be tuned by applying strain to design highly productive nanodevices. ${ }^{27}$ Therefore, it is of a great importance to investigate the mechanical behavior of silicene under applied strain.

Even though the pristine structure of silicene has a very high fracture strength, ${ }^{28,29}$ fracture toughness of silicene is significantly lower than most of the conventional engineering materials. $^{30}$ This offers a challenge in the fabrication of silicene based nanoelectronic devices. In general, fabrication of $2 \mathrm{D}$ materials involve various structural flaws such as defects and cracks. ${ }^{16,31,32}$ These inherent flaws significantly reduce the ultimate stress of the materials because of the high local stress concentration region. ${ }^{29,30,33,34}$ Numerous investigations have been conducted to probe the fracture mechanism of $2 \mathrm{D}$ nanosheets with pre-existing cracks. ${ }^{7,35}$ Specifically, the failure mechanism of single crystal and nanocrystalline silicene have been extensively studied ${ }^{\mathbf{3 0 , 3 6}}$ due to the challenge concerning the fabrication method. Yuan et al. ${ }^{29}$ investigated the mechanical properties of armchair silicene nanoribbons containing an edge crack using molecular dynamics (MD) simulations. The effects of edge crack length, strain rate, temperature on the fracture strength have been studied. Ding et al. ${ }^{33}$ investigated the effects 
of defects such as vacancies, crack and grain boundaries on the mechanical properties of silicene sheet. They found a linear decreasing relationship between the strength of silicene sheet and the defect parameters. From these studies, it is inevitable that the mechanical properties of silicene at nanoscale are significantly affected by the presence of pre-existing cracks. Therefore, it greatly necessitates to focus on finding an engineering solution to compensate for the decrement of mechanical properties. Previously, very few studies have been performed to elucidate various processes to increase the fracture strength of graphene by lattice trapping or crack arresting. These processes involve introduction of various topological defects in the structure ${ }^{37-41}$ which has a major drawback due to their inapplicability in the perfect lattices. ${ }^{19,20,42}$ Also, the fracture behavior of silicene is much different than graphene. Previously, Rakib et al. ${ }^{30}$ investigated the comparative mechanical properties and fracture toughness of graphene, silicene and hexagonal boron nitride. Chung et al. ${ }^{43}$ showed that unlike graphene, the stress in the silicene nanosheet is nonuniformly distributed due to the rearrangement of atoms in the silicene nanosheet via ring reorientation under applied strain. They also described the difference in crack propagation direction between silicene and graphene nanosheet.

In continuum fracture mechanics, it is well established that interactions of micro-cracks and voids with the crack can significantly alter the stress concentration around the crack tip. ${ }^{44,45}$ However, atomistic studies on multiple crack interaction are quite limited. Liu et al. ${ }^{46}$ investigated the crack void interaction in $\alpha$-Fe and Dewapriya et al. showed that lattice trapping of graphene can be achieved by placing holes and multiple cracks around the crack tip. ${ }^{47,48}$ However, to the best of our knowledge, no such study has been conducted to provide any solution to increase the mechanical properties of silicene nanosheet containing pre-existing crack.

Again, the crack propagation speed of other 2D materials such as graphene ${ }^{49}$ hexagonal boron nitride ${ }^{50}$ and $\mathrm{MoS}_{2}$ (ref. 51) have been investigated previously. But in case of silicene, to the best of our knowledge, no such study is done.

In this study, for the first time, a practically feasible way is presented to increase the ultimate stress of silicene nanosheet containing a main central crack by symmetrically placing auxiliary cracks around the crack tip. The enhancement of the ultimate stress is demonstrated by applying uniaxial load on silicene using MD simulation. In this work, we placed the auxiliary cracks in different positions and we found that these cracks can shield or amply the crack propagation in silicene nanosheet. The effects of temperature on the sheet containing auxiliary cracks are studied. The crack propagation speed of silicene and the effect of auxiliary crack on this speed is investigated. The size effects of auxiliary cracks are also studied for both armchair and zigzag loading condition. Finally, we analyzed the failure resisting and crack arresting phenomena for both armchair and zigzag loading conditions which provides new insights on the possibilities of strain engineering of silicene as well as adopting unique ways to improve the mechanical properties of silicene nanosheet which may lead to a better fabrication process of silicene based nanodevices.

\section{Methodology}

At first, a $300 \AA \times 300 \AA$ nanosheet is constructed for uniaxial tensile test simulation. Then cracks of different lengths (approximately $10 \AA, 20 \AA, 30 \AA, 40 \AA$ and $50 \AA$ ) are introduced either along armchair or zigzag direction of the nanosheet to investigate the effects of crack length on fracture strength of silicene. The cracks are created by deleting a few rows of atoms. Similar approach for creating initial cracks has been employed in previous studies. ${ }^{30,36,47,48}$ The size of nanosheet is taken such a way that the sample size effect remains insignificant while keeping computational time within reasonable limit. Note that, the crack lengths are chosen such that the length and width of the nanosheet are at least ten times larger than half crack length in order to avoid the effects of finite dimension..$^{52,53}$

In order to investigate the interaction between main crack and auxiliary cracks, one main crack at the center and four auxiliary symmetric cracks at different positions (Fig. 1) are introduced in the nanosheet. The size of the auxiliary cracks are kept equal to that of the main crack and they are always oriented perpendicularly to the direction of the main crack. Fig. 1 shows the relative position of auxiliary cracks and the main crack in cartesian and polar co-ordinate system. Because of the symmetry, only the co-ordinates of the auxiliary crack positioned in the first quadrant is used to define the positions of all the auxiliary cracks as shown in Fig. 1. To describe the atomistic phenomenon of fracture and investigate the mechanical properties, molecular dynamics simulations are used extensively for silicene. $^{28,30,33,36,54-61}$ All the simulations are performed in LAMMPS $^{62}$ software package where periodic boundary conditions were incorporated along the in plane directions $(x$ and $y$ ) of the sheet. In order to eliminate the effects of free edges, ${ }^{63}$ the size of the simulation box was taken as an exact multiple of a unit cell in both directions. The systems were first relaxed using conjugate gradient (CG) minimization scheme. Then NVE ensemble was used to equilibrate the systems which was followed by isothermal-isobaric (NPT) simulations at $300 \mathrm{~K}$ temperature and atmospheric pressure. Next, a constant strain rate of $10^{9} \mathrm{~s}^{-1}$ is applied along the $y$ direction which is relatively higher compared to the practical cases. However, such a high strain rate-allows to perform simulations with a reasonable computational resource-have been commonly used in atomistic scale simulations to investigate the material failure phenomena. ${ }^{30,36,64}$ Atomic stresses under deformation is described by the following equation ${ }^{65}$

$$
\sigma_{\text {virial }}(r)=\frac{1}{\Omega} \sum_{i}\left[\left(-m_{i} \dot{u}_{i} \otimes \dot{u}_{i}+\frac{1}{2} \sum_{j \neq i} r_{i j} \otimes f_{i j}\right)\right]
$$

where the sum is taken for all the atoms in the volume, $m_{i}$ denotes the mass of atom $i, \dot{u}_{i}$ is the time derivative of the displacement, $r_{i j}$ denotes the position vector and $f_{i j}$ denotes the interatomic force applied on atom $i$ by atom $j$. The thickness of the silicene nanosheet is taken as $3.13 \AA$.

In order to describe the interactions between silicon atoms in the nanosheet, an optimized Stillinger-Weber $(\mathrm{SW})^{66}$ potential is used. This potential is incorporated because of its 

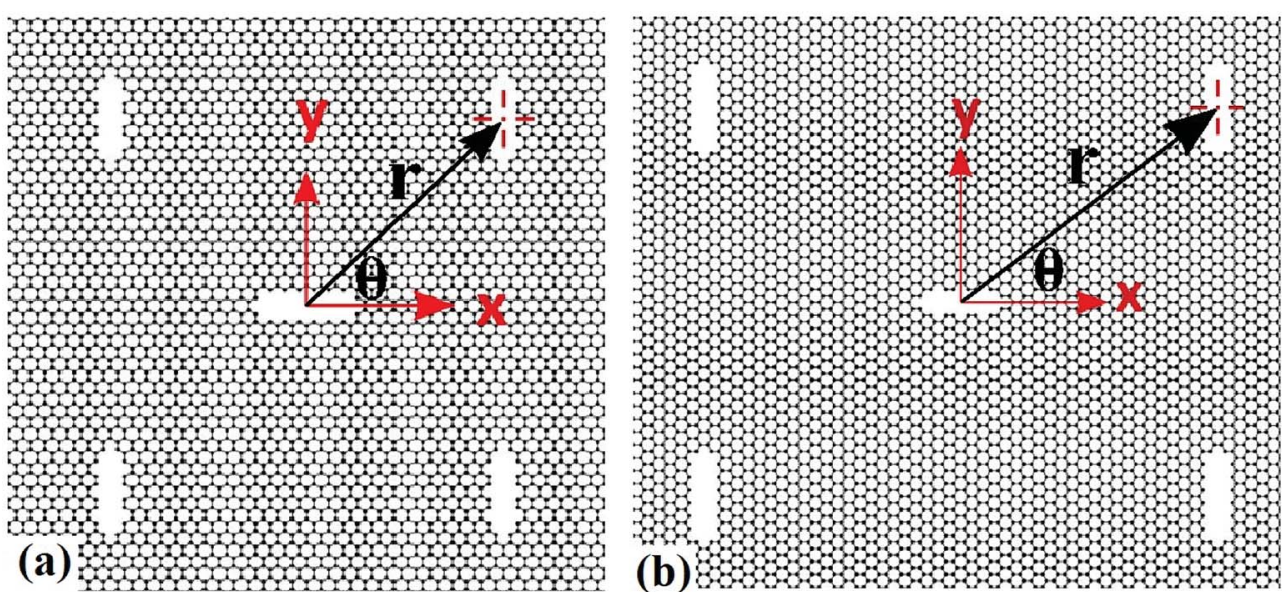

Fig. 1 Silicene nanosheets containing four auxiliary cracks and one main crack in (a) zigzag, (b) armchair direction. Uniaxial tension is applied along perpendicular direction to the main crack.

successful use in describing thermal ${ }^{67}$ and mechanical properties $^{30,36}$ of silicene previously. Furthermore, the obtained buckling height $(0.44 \AA)$ after relaxation is in good agreement with DFT calculations. ${ }^{68}$ The SW potential consists of a two body term and a three body term describing the bond stretching and bond breaking interactions respectively. The mathematical expressions of these interactions are described below-

$$
\begin{gathered}
\Phi=\sum_{i<j} V_{2}+\sum_{i<j<k} V_{3} \\
V_{2}=\varepsilon A\left(B \sigma_{a}^{p} r_{i j}{ }^{-p}-\sigma_{a}{ }^{q} r_{i j}{ }^{-q}\right) e^{\left[\sigma_{a}\left(r_{i j}-a_{1} \sigma_{a}\right)^{-1}\right]} \\
V_{3}=\varepsilon \lambda e^{\left[\gamma \sigma_{a}\left(r_{i j}-a_{1} \sigma_{a}\right)^{-1}+\gamma \sigma_{a}\left(r_{j k}-a_{1} \sigma_{a}\right)^{-1}\right]}\left(\cos \theta_{i j k}-\cos \theta_{0}\right)^{2} .
\end{gathered}
$$

Here, two-body and three-body terms are denoted by $V_{2}$ and $V_{3}$ respectively; $r_{i j}$ denotes the distance between atoms $i$ and $j ; \theta_{i j k}$ denotes the angle between bond $i j$ and $j k$; equilibrium angle between two bonds is denoted by $\theta_{0} ; A, B$, and other parameters are the coefficients required to fit while developing the potential. The values of these parameters can be found in the ref. 36 and 67.

In order to validate the current method, the fracture strength of a $300 \AA \times 300 \AA$ silicene nanosheet has been compared with the literature. ${ }^{30}$ Uniaxial tension is applied in either armchair or zigzag direction at $100 \mathrm{~K}$ temperature. The comparison between the values are shown in Table 1 . The close proximity of the values obtained by our simulation method and existing literature verifies our method and adopted potential to describe the interactions in silicene nanosheet.

Table 1 Comparison between values obtained by present method and existing literature ${ }^{30}$

\begin{tabular}{lllll}
\hline & \multicolumn{2}{l}{ Ultimate Stress (GPa) } & \multicolumn{2}{l}{ Ultimate Strain (\%) } \\
\hline Direction & Armchair & Zigzag & Armchair & Zigzag \\
Present study & 28.5 & 30.8 & 27.1 & 31 \\
Ref. 30 & 27.8 & 30.6 & 27 & 31
\end{tabular}

\section{Results and discussions}

\subsection{Stress-strain relationships for pre-cracked silicene}

We investigate the effects of central crack length on the ultimate stress of silicene nanosheet. Fig. 2 describes the effect of crack length on the ultimate stress of silicene nanosheet containing a central crack. It is evident from the figure that higher crack length reduces the ultimate stress and material failure occurs at relatively lower strain values for both armchair and zigzag loading conditions. This phenomena is quite expected as higher crack length induces more localized stress around the crack tip. ${ }^{69,70}$ We observe that, for the same crack lengths, the armchair loading direction fails at lower ultimate strains compared to the zigzag loading direction. This phenomenon is observed and explained in previous studies. ${ }^{28,30}$ In the case of armchair loading direction, one third of the total bonds are parallel to the loading direction. So, the local bond strain in silicon atoms is proportional to the global strain and failure occurs at such global strain when the stretched bond length exceeds the value of critical bond length. However, in case of zigzag loading direction, the bonds are at either $30^{\circ}$ or $90^{\circ}$ angle with the loading direction. Hence, to reach the critical bond length, much larger stretching of the bond is needed resulting in much higher global strain. So, properties are slightly better when the silicene nanosheet is loaded in zigzag direction.

\subsection{The effects of auxiliary cracks}

3.2.1. Effects of spatial position of auxiliary cracks. Study focusing on the effects of spatial positions of the auxiliary cracks on the fracture strength is necessary as some positions may trigger the ultimate failure whereas other positions may shield the fracture of the material. The length of auxiliary crack is kept equal to the main crack length for both armchair and zigzag loading conditions in the simulations. The variation of ultimate stress with the position of auxiliary cracks is shown in Fig. 3. From Fig. 3(a), it is evident that in case of loading along armchair direction, the larger the distance of the auxiliary cracks from the main crack at a definite $\theta=76.7^{\circ}$, the least the shielding effect is. For this case, at 


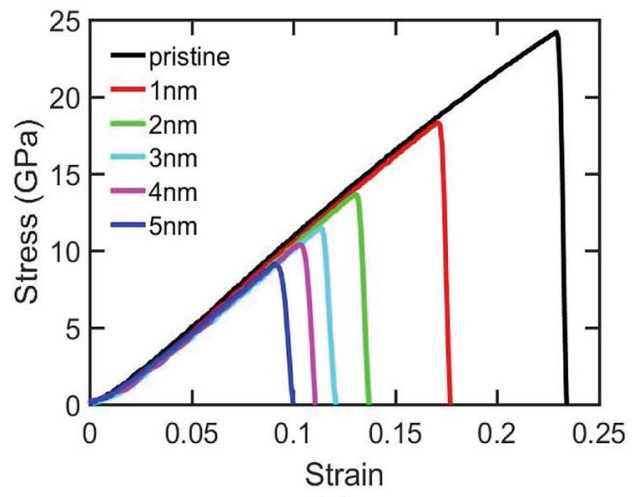

(a)

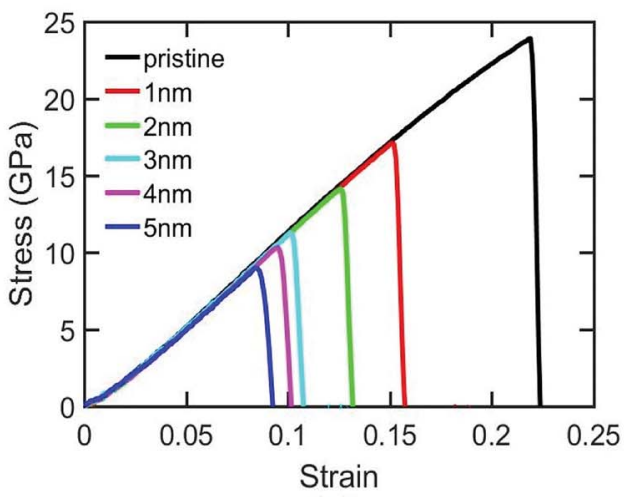

(b)

Fig. 2 Stress-strain relationships for silicene nanosheet containing a central crack of different lengths. Uniaxial tensile load is applied in (a) zigzag direction, (b) armchair direction.

a value of $r>60 \AA$, the auxiliary cracks assists the failure rather than shielding while the failure shielding phenomenon is more prominent for $r<60 \AA$. This can be explained by the crack shielding mechanism of the auxiliary cracks. Crack shielding occurs when the crack tip stress is reduced. A small area around the tips of the auxiliary cracks remain unstressed because of the parallelism of them with the loading direction. For a lower value of $r$, this unstressed tip area of the auxiliary cracks remains in close proximity to the central crack tips. Thus, this unstressed zone is superimposed on the highly stressed zone of the central crack tips and reduce the tip stress. However, if the distance between the central crack and auxiliary crack gets larger, the unstressed zone of the auxiliary cracks is not superimposed on the main crack tip and the sheet fails at a lower stress. From Fig. 3(b), we observe that the ultimate stress is the highest for $\theta=43.4^{\circ}$ in the case of armchair loading direction. The reason is the same as stated earlier. For this position of auxiliary crack, the unstressed zone produced by the auxiliary cracks is imposed on the high stressed zone of the central crack leading to a mitigation of the stress concentration at the central crack tips.

Interestingly, for zigzag loading condition, the shielding effects are very prominent due to a unique bond rotation phenomenon at the crack tip leading to the formation of a SW defect at the tip and thus reducing the tip stress (see ESI Video. $\dagger$ The rotation of bond is indicated by the red atoms). This type of atomic fracture process involving the formation of SW defect have also been observed earlier for graphene ${ }^{71}$ and silicon. ${ }^{72}$ This unique phenomenon does not occur without the presence of auxiliary cracks. However, the rotation of the bond is facilitated if the atomic movements at the tips are directed towards the loading direction ${ }^{73}$ which only happens in case of zigzag loading.

Fig. 4 depicts the displacements of the atoms near the crack tips for different positions of auxiliary cracks from their initial unstressed condition at about $9 \%$ strain. Because of the presence of the auxiliary cracks, the atomic movements change as shown in Fig. $4(\mathrm{~b})$ and (c). For $\theta=76.7^{\circ}$ and $r=43.6 \AA$, atomic movements are parallel (Fig. 4(c)) to the loading direction facilitating the bond rotation process.

Furthermore, the SW defect formation is found to be independent of the value of $r$. For this reason, for $\theta=76.7^{\circ}$, SW defect is formed near the tip irrespective of $r$ which increases the ultimate stress (Fig. 3(a)). The slight variations of the ultimate stresses in Fig. 3(a) are due to the time of evolution of the

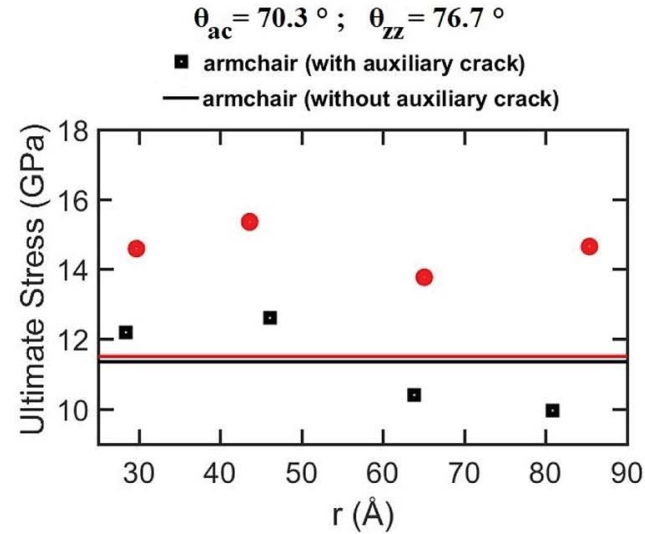

(a)

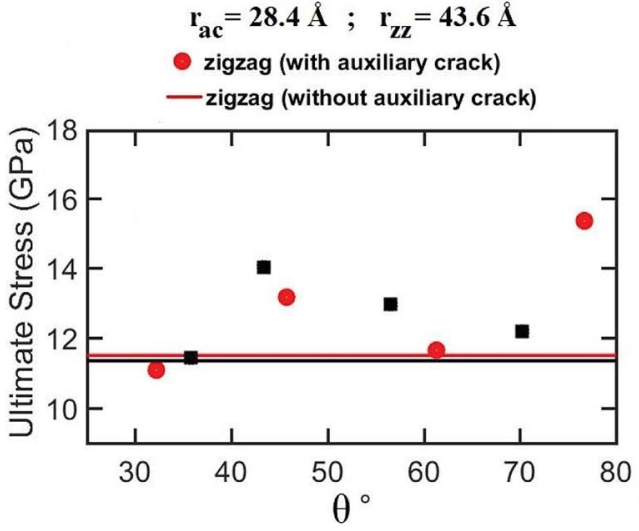

(b)

Fig. 3 Variation of ultimate stress in the cracked silicene nanosheet with (a) distance ( $r$ ) and (b) angle $(\theta)$ of the auxiliary crack. 


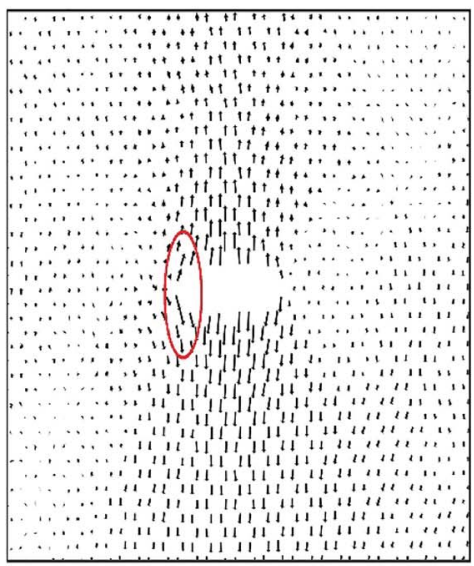

(a)

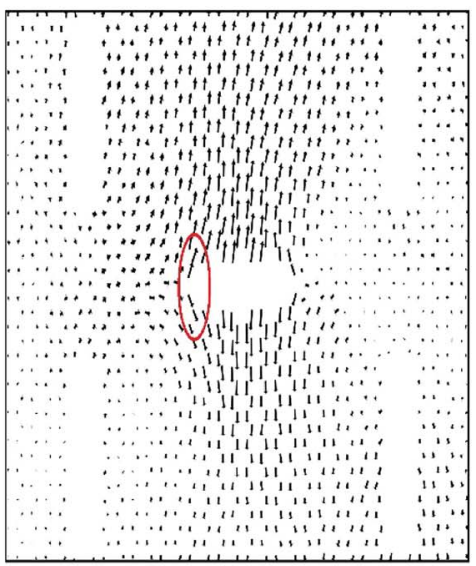

(b)

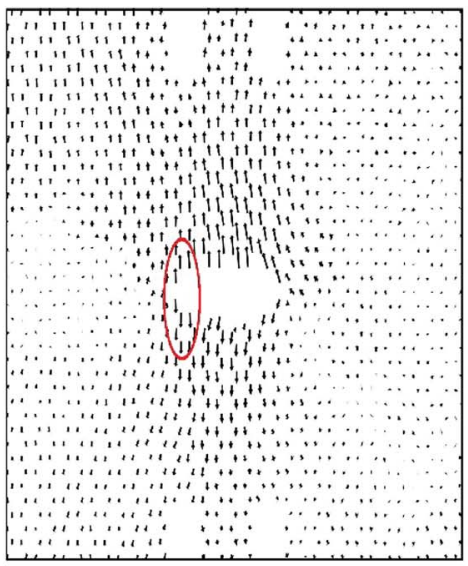

(c)

Fig. 4 Atomic movements around the crack tip depicted by displacement vectors at $9 \%$ strain for silicene nanosheet containing (a) no auxiliary crack, (b) auxiliary crack at $r=43.6 \AA$ and $\theta=32.2^{\circ}$, (c) auxiliary crack at $r=43.6 \AA$ and $\theta=76.7^{\circ}$. For (a) and (b), atomic movements at left crack tip are at an angle with the loading direction which impedes the bond rotation whereas for (c), atomic movements are parallel to the loading direction facilitating the rotation of the bond at the crack tip.

defect. If the defect is formed at a lower strain, the ultimate stress is higher and vice versa. On the contrary, the SW defect formation is highly dependent on the angular position of the auxiliary cracks. Fig. 3(b) illustrates the dependency of the ultimate stress on the variation of angular position of the auxiliary cracks. For $\theta=32.2^{\circ}$ and $\theta=61.3^{\circ}$, the pattern of the atomic movements are at an angle to the direction of loading (Fig. 4(b)) which impedes the rotation of the bond at the crack tip and ultimate stress becomes lower. This mechanism is further explained in Section 3.3.2.

3.2.2. Effects of auxiliary crack length. To investigate the effects of auxiliary crack length on the fracture strength of silicene nanosheet, the position of the auxiliary cracks is chosen as $r=28.4 \AA$ and $\theta=70.3^{\circ}$ for armchair loading and as $r=43.6 \AA$ and $\theta=76.7^{\circ}$ for zigzag directional loading conditions. Fig. 5 shows the variation of ultimate stress with the change of auxiliary crack length for both armchair and zigzag loading conditions. From this figure, it is clear that in case of zigzag loading, fracture strength is higher compared to that of the armchair loading condition. This is because of the SW defect formation phenomenon at the crack tips for the zigzag loading condition as discussed in the previous section. For the case of zigzag loading, the effect of auxiliary crack size on the ultimate stress seems random. With the change of the auxiliary crack length, the stress trajectory and the relative position of the main crack tip and auxiliary crack tips are changed. These phenomena result in a difference in the strain values at which the defect formation occurs. For this reason, a direct relation between the auxiliary crack length and ultimate stress cannot be observed. However, irrespective of the crack length, the ultimate stress is much higher compared to the one without any auxiliary crack.

For armchair loading direction, ultimate stress is the lowest for the lowest crack length. Because at a particular position, the lower the crack length, the higher the distance between the tips of main crack and auxiliary crack. Thus, the unstressed zone ahead of the auxiliary crack tip remains smaller and cannot provide enough shielding for the main crack and failure

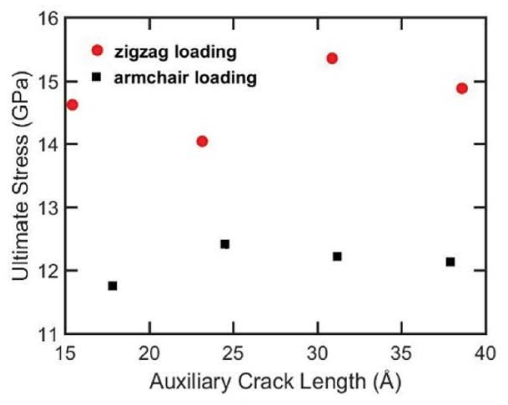

(a)

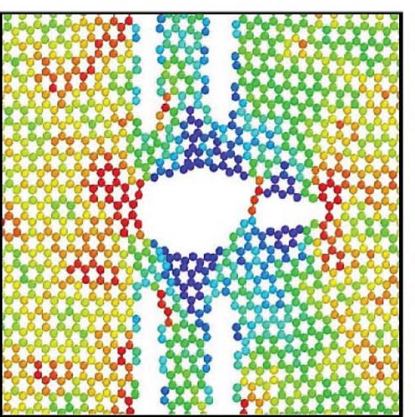

(b)

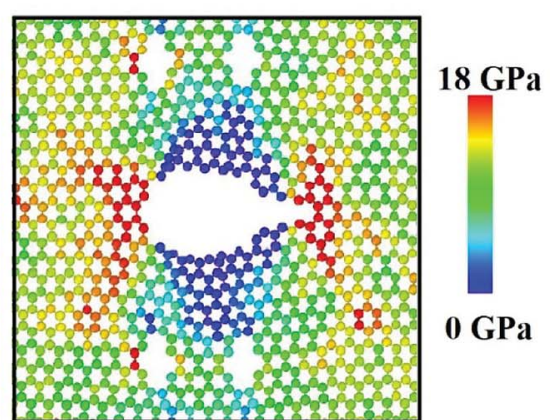

(c)

Fig. 5 (a) Variation of ultimate stress with the variation of the length of the auxiliary cracks $\left(r=28.4 \AA\right.$ and $\theta=70.3^{\circ}$ for armchair and $r=43.6 \AA$ and $\theta=76.7^{\circ}$ for zigzag loading cases). Failure initiation for the cases of (b) largest and (c) smallest auxiliary crack length. Because of the high plasticity, failure occurs ahead of the main crack tip for the largest auxiliary crack length. 


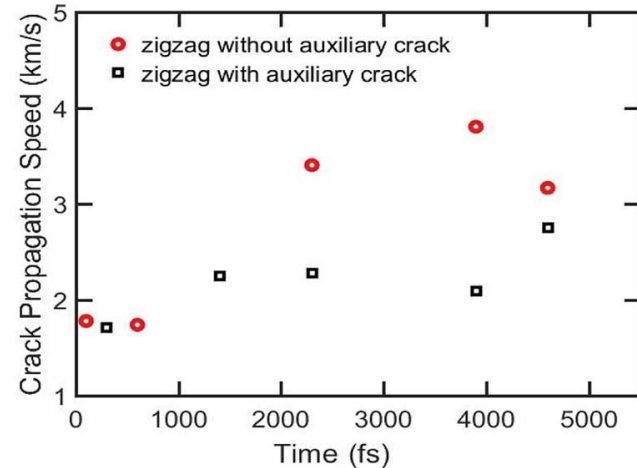

(a)

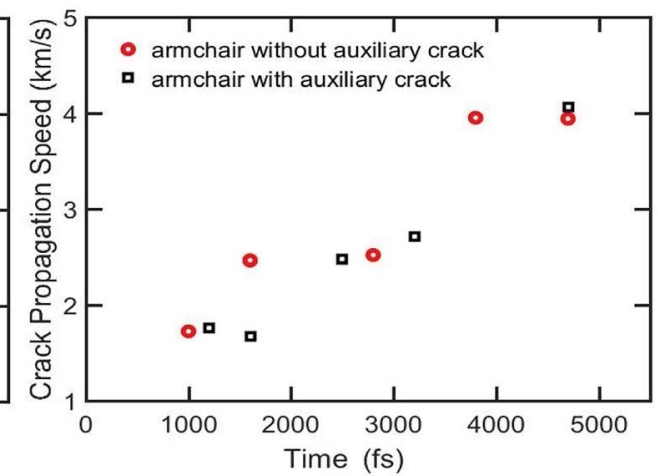

(b)

Fig. 6 Variation of crack propagation speed for pre-cracked silicene nanosheet loaded in (a) zigzag and (b) armchair direction with the variation of time. Time is counted after the initiation of crack propagation.

initiates from the crack tip (Fig. 4(c)). However, if the auxiliary crack length gets too large, plastic deformations becomes more pronounced ahead of the central crack tip and failure initiates ahead of the crack tip rather than at the tip because of the high plasticity (Fig. 5(b)).

3.2.3. Effects of auxiliary cracks on the crack propagation speed. We investigated the effects of auxiliary cracks on the crack propagation speed. For this study, the position of the auxiliary cracks is chosen as $r=28.4 \AA$ and $\theta=70.3^{\circ}$ for loading along armchair direction and as $r=43.6 \AA$ and $\theta=76.7^{\circ}$ for loading along zigzag direction. The crack propagation speed is determined by dividing the crack tip advancement with the time taken. Fig. 6 shows the variation of crack tip speed for silicene nanosheet with time. Crack speed varies from about $1 \mathrm{~km}^{-1}$ to 4 $\mathrm{km}^{-1}$ at different time. However, with the presence of auxiliary cracks, the crack propagation velocity is much reduced for both armchair and zigzag loading conditions. This is due to the shielding nature of the auxiliary cracks which impedes the propagation of the main crack.

3.2.4. Effects of temperature. Fig. 7 shows the effects of temperature on the fracture strength of silicene nanosheet containing auxiliary crack positioned at $r=28.4 \AA$ and $\theta=70.3^{\circ}$ in case of loading along armchair direction and at $r=43.6 \AA$ and $\theta=76.7^{\circ}$ for zigzag loading condition. From the Fig. 7(a), it is clear that fracture strength decreases as the temperature increases. This phenomena is quite expected as higher temperature induces larger fluctuations in the chemical bond length between the atoms as well as enlarges the initial bond length..$^{59}$

In case of zigzag loading condition, MD simulation results shows interesting phenomena. It is clear from the figure that fracture strength increases with temperature upto $300 \mathrm{~K}$ temperature and then decreases. This is due to the crack shielding mechanism of silicene nanosheet. The crack propagation at $300 \mathrm{~K}$ temperature is mainly suppressed by the rotation of bonds at the crack tips. At this temperature, instead of bond breaking, this bond rotation phenomena occurs due to the energetic and kinetic favorability. ${ }^{71}$ On the other hand, at lower temperature, the bond rotation phenomenon is not favored due to the lower energy available. Therefore, bond breaking occurs which leads to failure at a lower ultimate stress. However, with the increase of temperature above $300 \mathrm{~K}$, the fracture strength of the sheet decreases again despite of the occurrence of bond rotation process. This is due to the higher atomic movements at these elevated temperatures leading to higher fluctuation in the bond length. ${ }^{59}$ Therefore, some of the

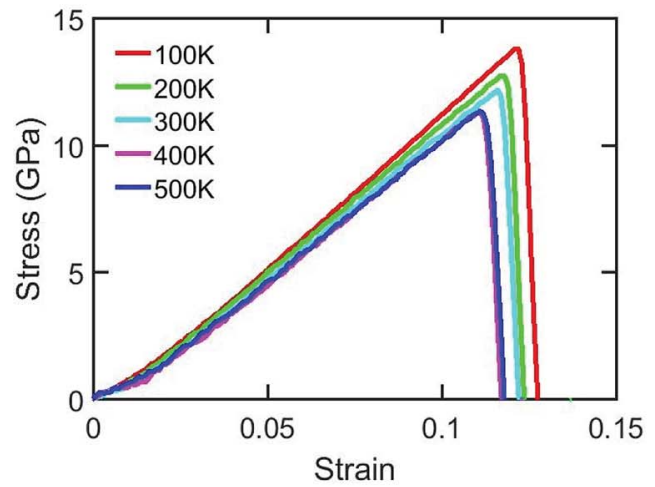

(a)

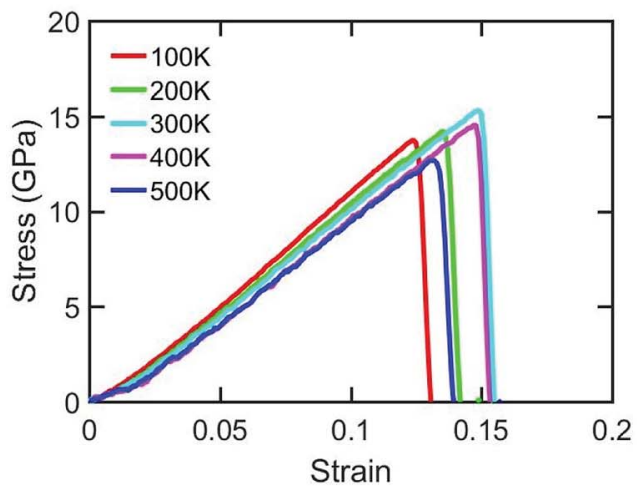

(b)

Fig. 7 Variation of fracture strength of silicene nanosheet containing auxiliary cracks with the variation of temperature and loaded in (a) armchair, (b) zigzag direction. 


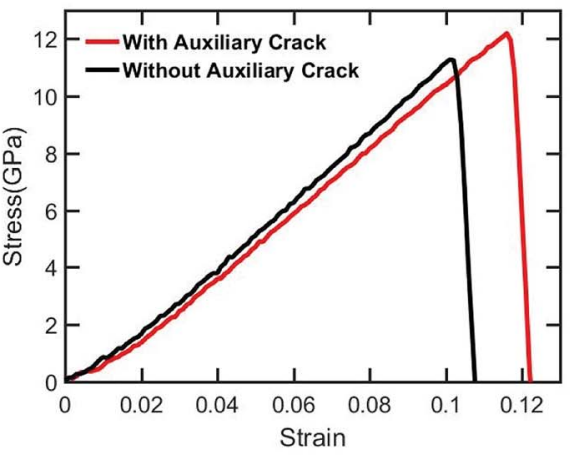

(a)

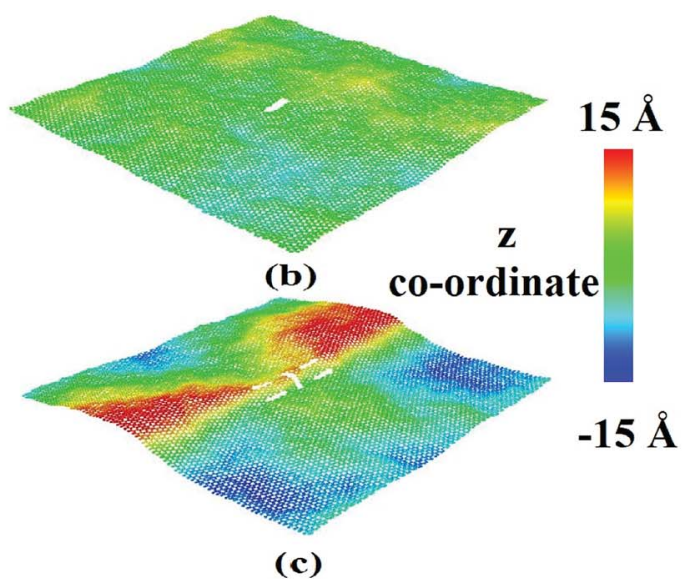

Fig. 8 (a) Stress-strain curves for nanosheets containing a central main crack with auxiliary cracks and without auxiliary cracks. Out of plane deformation of the sheet (b) without auxiliary crack and (c) with auxiliary cracks after relaxation. This out of plane deformation is responsible for the decrement of Young's modulus.

bond lengths exceed the critical bond length and material failure occurs at a lower ultimate stress and strain.

\subsection{Crack arresting mechanism}

3.3.1. Armchair loading. We analyze the mechanism of crack shielding phenomena provided by the auxiliary cracks positioned at $r=28.4 \AA$ and $\theta=70.3^{\circ}$ in case of armchair loading condition. Fig. 8(a) illustrates the comparison of the stress-strain curve between the nanosheets with and without the auxiliary cracks. From Fig. 8(a), it is clear that because of the presence of the auxiliary cracks, both the ultimate stress and strain increase while the Young's modulus decrease. With the presence of auxiliary cracks, the nanosheet displays significant out of plane movement (approximately 31.1 $\AA$ ) after relaxation whereas the nanosheet without auxiliary crack displays very low out of plane movement (approximately $14.5 \AA$ ) as shown in Fig. 8(b) and (c). The out of plane deformation is measured by the highest distance between the $z$ co-ordinates of two atoms in the sheet. The presence of the auxiliary cracks introduce more free edges in the nanosheet. ${ }^{74}$ After relaxation, this free edges lead to an edge energy and compressive edge force ultimately resulting in wrinkles in the sheet. ${ }^{75,76}$ With the applied load, at first, the wrinkles get flattened out and then the bonds start to (a)

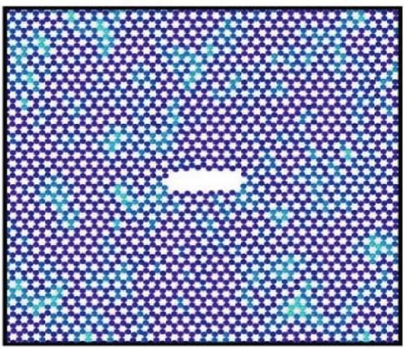

$\mathcal{E}=1 \%$

(b)

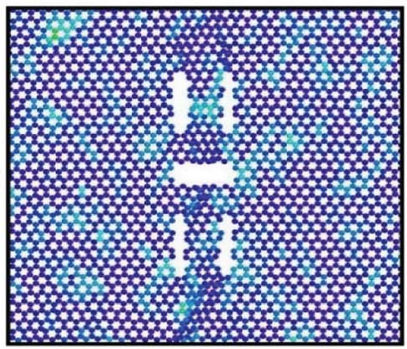

$\varepsilon=1 \%$

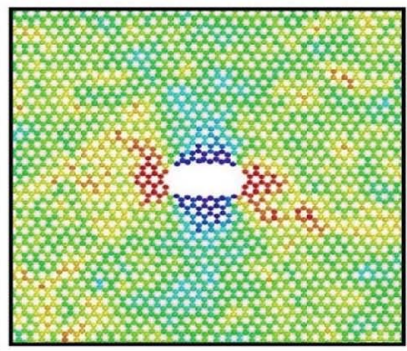

$\mathcal{E}=10 \%$

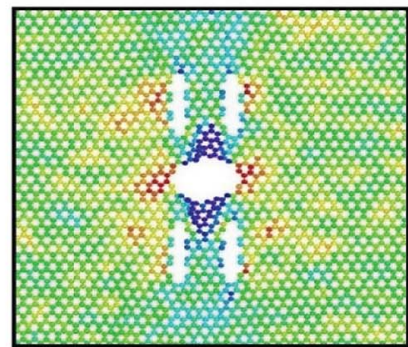

$\varepsilon=10 \%$

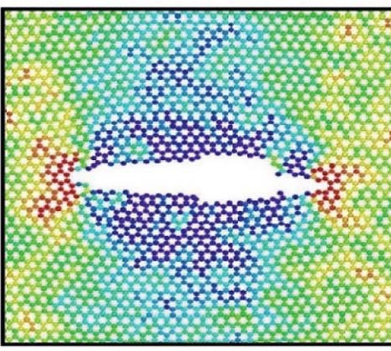

$\varepsilon=10.4 \%$

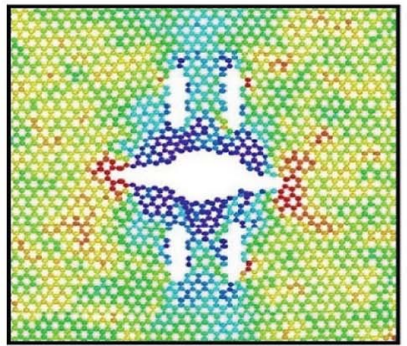

\section{GPa}

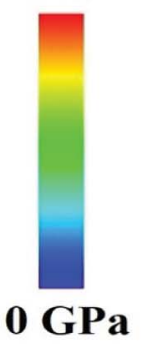

Fig. 9 Stress distribution at different strain depicting the failure mechanism of silicene nanosheets containing a main central crack of $3 \mathrm{~nm}$ length (a) without auxiliary crack; (b) with auxiliary cracks and loaded in armchair direction. Stress trajectory changes due to the presence of auxiliary cracks and reduces the density of stress at the central crack tips leading to higher ultimate stress. 
get stretched. Thus this deformation is responsible for the decrement of Young's modulus.

To elucidate the reason behind the increment of the ultimate stress of the sheet, the comparative stress distribution of the silicene nanosheet with and without auxiliary cracks are shown in Fig. 9 at different strains. As the instantaneous stress of an atom fluctuates very rapidly, ${ }^{65}$ time averaging is needed to gain the stress distribution of the nanosheet at any instant. ${ }^{47,48}$ To illustrate the stress distribution at a particular instant, we averaged the stresses of every atom for the last 100 timesteps to show the stress distribution. Fig. 9(a) shows the failure mechanism of the silicene nanosheet containing a 30 A central crack. From the figure, one can see that stress accumulation near the crack is the primary reason of failure for pre-cracked silicene nanosheet. At about $10 \%$ strain, the stress of the crack tip begins to exceed the maximum stress needed to break the $\mathrm{Si}-\mathrm{Si}$ bond which is reported as $25 \mathrm{GPa} \cdot{ }^{30}$ However, presence of the auxiliary cracks significantly alters the stress distribution around the crack tip due to the interaction between main and auxiliary cracks. The stress accumulation is also higher without auxiliary cracks than that with auxiliary cracks. Although the configuration of the main crack is kept same for both the cases, plastic deformation is more prominent in the latter case at higher strain due to the presence of the auxiliary cracks at such close proximity with the main crack ultimately leading to a change in the crack configuration. This plastic deformation enlarges the width as well as blunts the tips of the central crack. This type of crack configuration disfavors the higher stress concentration around the tips and failure is restricted until higher strain is applied.

3.3.2. Zigzag loading. We also investigate the shielding mechanism provided by the auxiliary cracks located at $r=43.6$ $\AA$ and $\theta=76.7^{\circ}$ for zigzag directional loading. From Fig. 3 , it is evident that ultimate stresses of silicene are higher for this case than the armchair loading. Fig. 10 shows the comparison of stress distribution between the silicene nanosheets with auxiliary cracks and without auxiliary cracks at different strains in case of loading in the zigzag. Without the presence of the auxiliary cracks, stress accumulates more at the crack tip under applied loading. With higher strains, stress accumulation at the crack tips gets higher and finally crack starts to propagate at about $11.4 \%$ strain (Fig. 10(a)). However, the presence of auxiliary cracks significantly alters failure phenomenon by following a unique defect formation. The presence of the auxiliary cracks at this particular position significantly influences the atomic movement near the tip. It has been shown in Fig. 4(c) that the atoms at the left tip is displaced almost parallel to the loading direction which in turns, facilitates the rotation of the bond adjacent to the left crack tip and forms a SW defect at about $10 \%$ strain. $^{73}$

This mechanism can be further explained with the help of Fig. 11 and 12 which show the variation of average atomic stress of the crack tip atoms at the left with the increase of global strain in silicene nanosheet without and with auxiliary cracks. The atomic snapshots around the crack are also shown at different strain levels. Initial atomic structure at the crack tip (a)

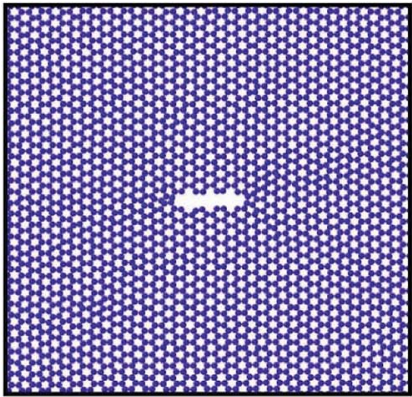

$\varepsilon=0 \%$

(b)

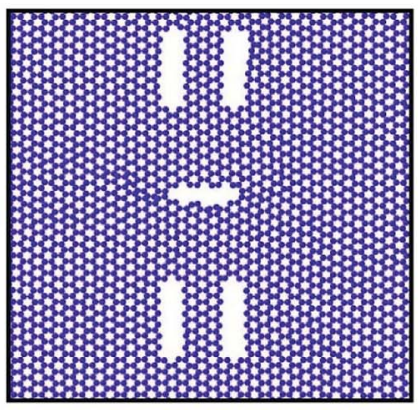

$\varepsilon=0 \%$

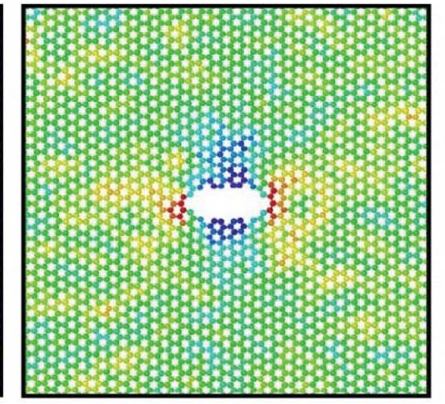

$\varepsilon=10.10 \%$

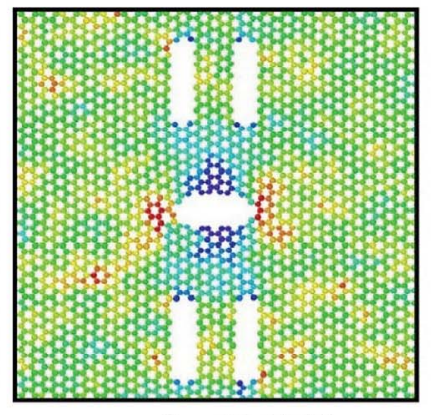

$\varepsilon=10.10 \%$

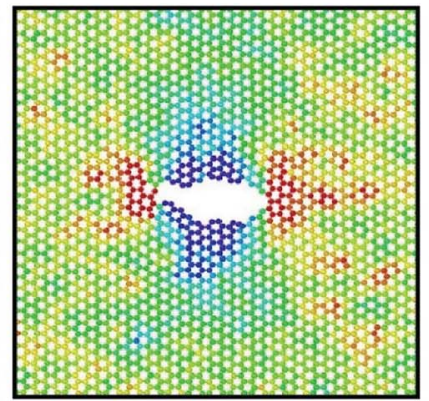

$\varepsilon=11.38 \%$

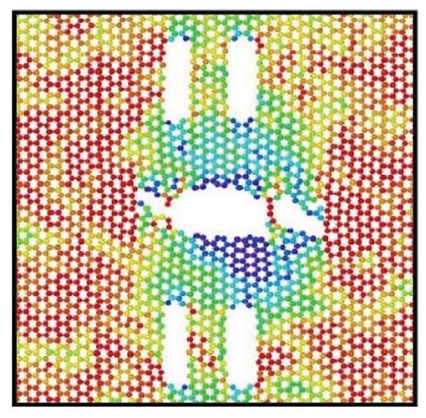

$\mathcal{E}=15.01 \%$
$20 \mathrm{GPa}$

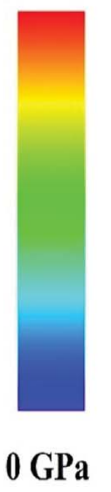

Fig. 10 Stress distribution at different strain depicting the failure mechanism of silicene nanosheets containing a main central crack of $3 \mathrm{~nm}$ length (a) without auxiliary crack; (b) with auxiliary cracks and loaded in zigzag direction. Bond rotation occurs at the crack tip due to the presence of auxiliary cracks at about $10 \%$ strain and arrest the propagation of crack. 


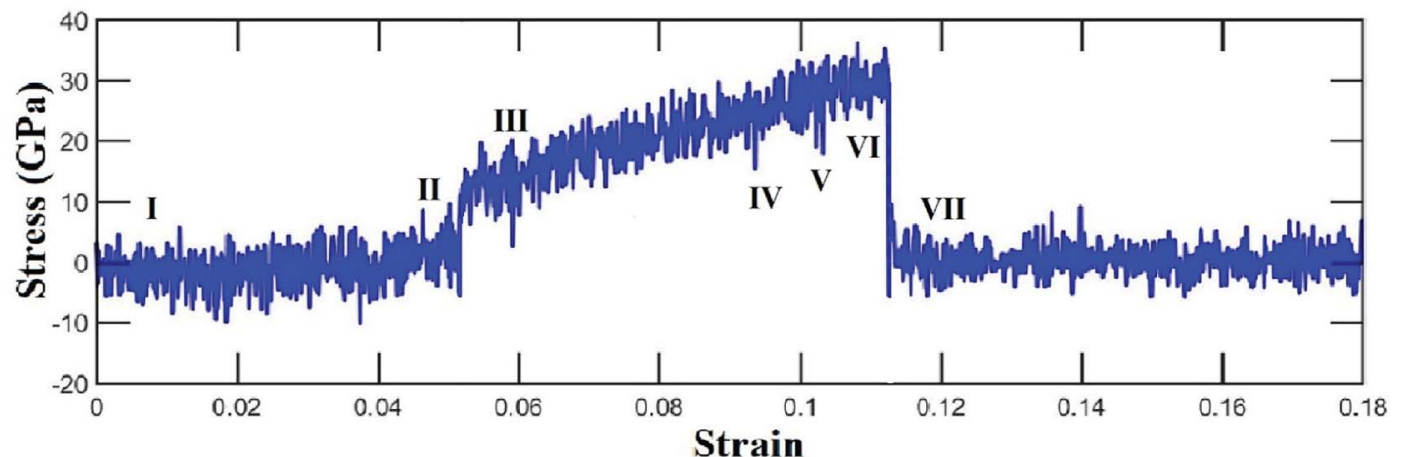

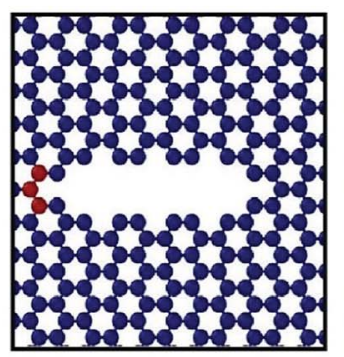

Initial configuration

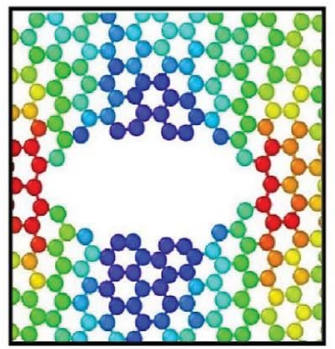

IV

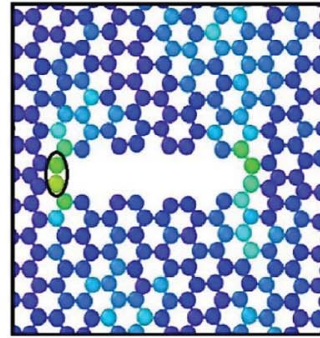

I

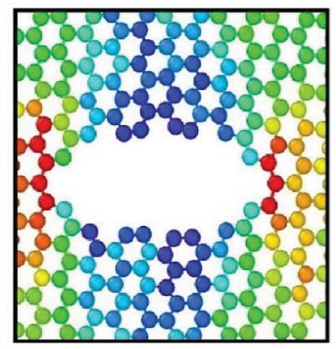

V

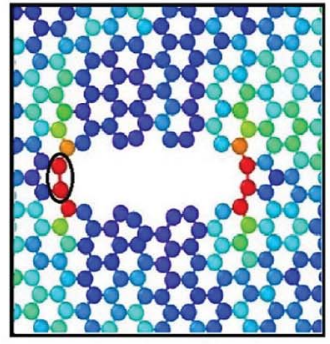

II

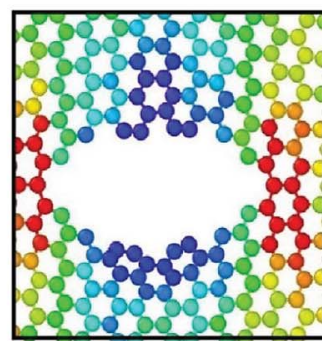

VI

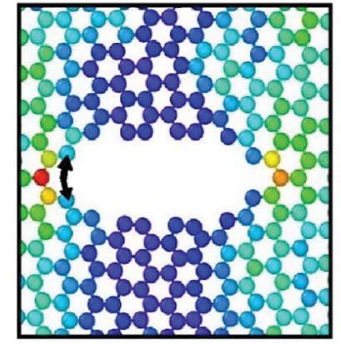

III

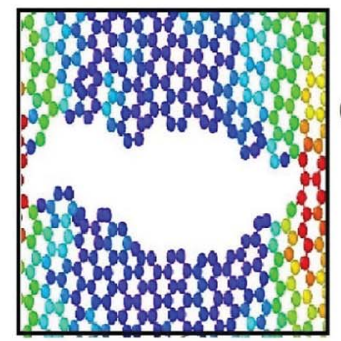

VII
$20 \mathrm{GPa}$

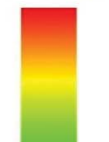

$0 \mathrm{GPa}$

Fig. 11 Variation of stress of crack tip atoms at the left with the increase of global strain without the auxiliary cracks. The tip atoms are depicted by the red circles shown in the initial configuration. (I) to (VII) depicts the stress distribution around the crack at various significant strain values. Weak bond created between the atoms adjacent to the tip gets broken at about $5 \%$ global strain as shown in (II) and (III).

changes after relaxation by the formation of a weak bond adjacent to the tip as shown in Fig. 11(I). For this reason, the tip atoms do not get stressed at this stage until the breaking of this weak bond as shown in Fig. 11(I) and (II). Sudden jump of the tip stress is observed at about $5 \%$ strain which corresponds to the breaking of the weak bond (Fig. 11(III)). The tip stress increases with the increase of global strain and finally failure initiates by the crack propagation as shown in Fig. 11(IV)-(VII).

In Fig. 12, it is observed that a weak bond near the tip is formed and atomic stress increase with a sudden jump because of the rupture of the weak bond with the applied strain in a similar way as in the case without auxiliary crack. At higher strain, the bond rotation occurs near the tip which reduces the stress of the tip atoms as shown in Fig. 12(V). Similar occurrences are observed for right crack tip as well. As a result, comparatively higher strain is required to initiate the failure of the material (Fig. 12(VI)). The reason of this bond rotation is behind the alteration of the pattern of the atomic movements. Atomic movements becomes parallel to the loading direction when the strain exceeds about 5\%. This type of displacement of atoms has the ability to sustain both bond rotation and bond stretching process. ${ }^{73}$ Previously, it is reported that two competing process of fracture, either bond rotation or bond breaking at the tip, can occur during the fracture process depending on the energetic and kinetic favorability. ${ }^{71}$ It is also reported for graphene nanosheet and nanotube that the formation of defect is more energetically favorable at higher strain as this configuration can reduce tensile strain due to the ability of heptagonal configuration of bonds to be stretched more than hexagonal configuration. ${ }^{77,78}$ The atomic movements at the crack tip are found to be parallel with the loading direction whereas the movement is at an angle without the presence of auxiliary cracks (Fig. 4). This type of atomic movement accompanied by high strain kinetically and energetically favors the bond rotation process. For this reason, the fracture strength of silicene becomes more enhanced by the presence of auxiliary cracks in case of zigzag loading. 


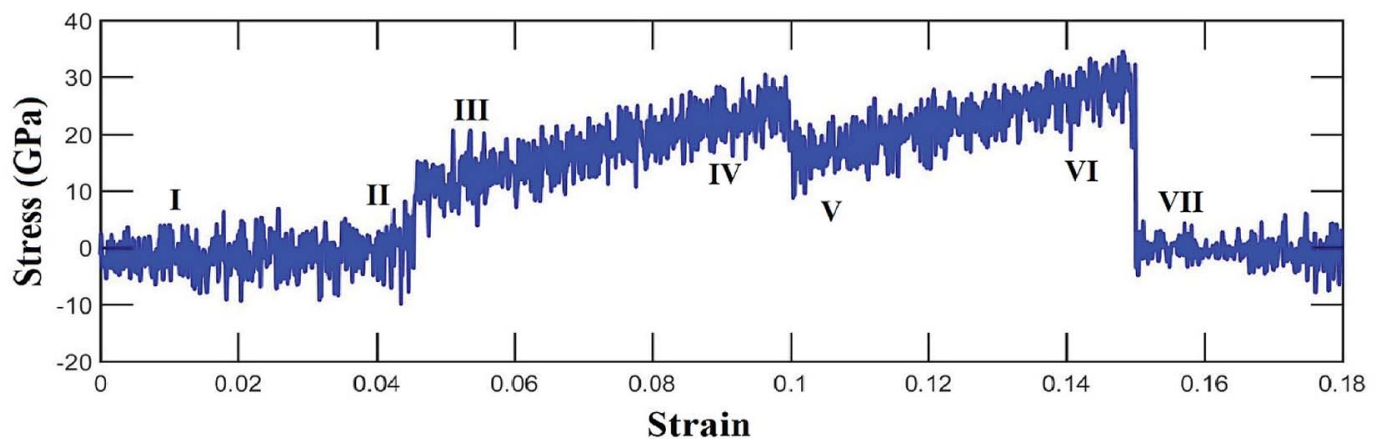

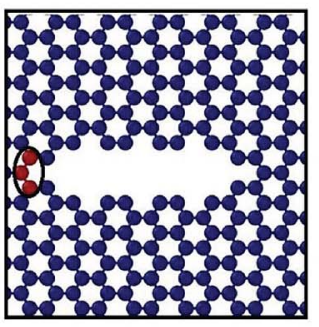

Initial configuration

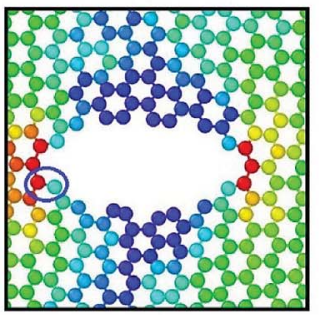

IV

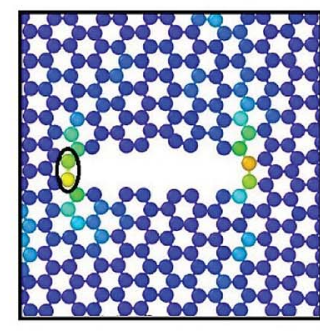

I

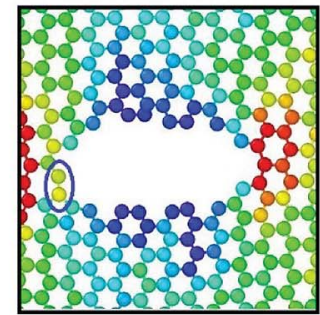

V

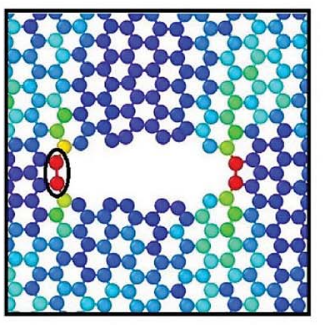

II

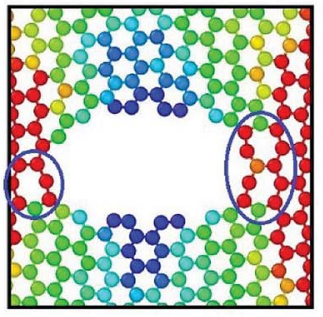

VI

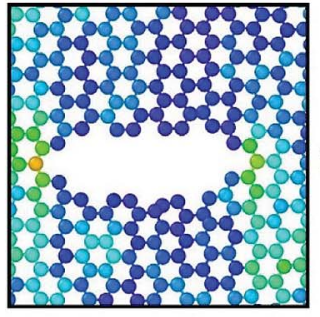

III

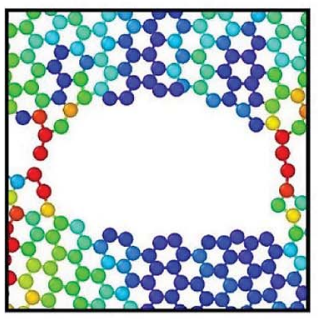

VII
$20 \mathrm{GPa}$

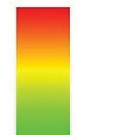

0 GPa

Fig. 12 Variation of left tip atoms' stress with the increase of global strain in the presence of auxiliary cracks at $r=43.6 \AA$ and $\theta=76.7^{\circ}$. The red marked atoms in the initial configuration and the black circles in I and II indicates left tip atoms. (I) to (VII) depicts the stress distribution around the crack at various significant strain values. Weak bond created between the atoms adjacent to the tip gets broken at about $5 \%$ global strain as shown in (II) and (III). (IV) and (V) depicts the rotation of atomic bond adjacent to the crack tip highlighted by the blue circle at about $10 \%$ strain. The stress concentration near the crack tip at the left decreases by this phenomenon. Furthermore, (VI) shows the formation of these kinds of defects at both crack tips. Initiation of failure at about $15 \%$ strain is depicted by (VII).

\section{Conclusion}

In this research, atomistic simulations have been carried out to investigate the effects of auxiliary cracks on the fracture strength of silicene. It has been observed that the fracture strength of silicene nanosheet in both loading directions increases significantly with the incorporation of auxiliary cracks, and it is more prominent in case of zigzag loading direction than the armchair loading direction. The crack propagation speed and the effects of temperature on the silicene nanosheet containing auxiliary cracks are also studied. The fracture strength shows an interesting increasingdecreasing trend with the increase of temperature. Moreover, in case of zigzag loading direction, for some specific locations of the auxiliary cracks, the atomic movements at the central crack tips have been observed parallel to the loading direction which leads to the occurrence of a bond rotation at the crack tip that in turn increases the fracture strength of the silicene nanosheet significantly. It is also observed that the presence of the auxiliary cracks invokes out of plane deformation of the silicene sheet. Finally, this study sheds light on understanding the failure mechanism of silicene nanosheet more extensively. Also this study opens a new possibility for tailoring the fracture strength of silicene nanosheet. Again the increased resistance to fracture may lead to possible strain engineering of silicene. Alongside the improvement of mechanical properties and possibility of strain engineering, the bond rotation due to the applied strain along zigzag direction might enable the alteration of local electronic properties of silicene nanosheet which may lead to noble applications.

\section{Conflicts of interest}

There are no conflicts to declare.

\section{Acknowledgements}

The authors of this paper would like to express their gratitude to the Department of Mechanical Engineering, BUET for providing the computational facilities. They would also like to 
acknowledge Multiscale Mechanical Modelling and Research Network (MMMRN) group for the technical support to conduct the research.

\section{References}

$1 \mathrm{X}$. Gu and R. Yang, arXiv preprint arXiv:1509.07762.

2 B. Zhu, X. Chen and X. Cui, Sci. Rep., 2015, 5, 9218.

3 H.-P. Komsa and A. V. Krasheninnikov, Phys. Rev. B, 2012, 86, 241201.

4 M. M. Ugeda, A. J. Bradley, S.-F. Shi, F. H. da Jornada, Y. Zhang, D. Y. Qiu, W. Ruan, S.-K. Mo, Z. Hussain, Z.-X. Shen, F. Wang, S. G. Louie and M. F. Crommie, Nat. Mater., 2014, 13, 1091-1095.

5 T. Borzda, C. Gadermaier, N. Vujicic, P. Topolovsek, M. Borovsak, T. Mertelj, D. Viola, C. Manzoni, E. A. A. Pogna, D. Brida, M. R. Antognazza, F. Scotognella, G. Lanzani, G. Cerullo and D. Mihailovic, arXiv:1412.5338 [cond-mat].

6 A. K. Geim and K. S. Novoselov, Nat. Mater., 2007, 6, 183-191. 7 D. Akinwande, C. J. Brennan, J. S. Bunch, P. Egberts, J. R. Felts, H. Gao, R. Huang, J.-S. Kim, T. Li, Y. Li, K. M. Liechti, N. Lu, H. S. Park, E. J. Reed, P. Wang, B. I. Yakobson, T. Zhang, Y.-W. Zhang, Y. Zhou and Y. Zhu, Extreme Mech. Lett., 2017, 13, 42-77.

8 J. A. Jackman, N.-J. Cho, M. Nishikawa, G. Yoshikawa, T. Mori, L. K. Shrestha and K. Ariga, Chem.-Asian J., 2018, just accepted.

9 A. Gupta, T. Sakthivel and S. Seal, Prog. Mater. Sci., 2015, 73, 44-126.

10 G. R. Bhimanapati, Z. Lin, V. Meunier, Y. Jung, J. Cha, S. Das, D. Xiao, Y. Son, M. S. Strano, V. R. Cooper, L. Liang, S. G. Louie, E. Ringe, W. Zhou, S. S. Kim, R. R. Naik, B. G. Sumpter, H. Terrones, F. Xia, Y. Wang, J. Zhu, D. Akinwande, N. Alem, J. A. Schuller, R. E. Schaak, M. Terrones and J. A. Robinson, ACS Nano, 2015, 9, 1150911539.

11 S. Z. Butler, S. M. Hollen, L. Cao, Y. Cui, J. A. Gupta, H. R. Gutiérrez, T. F. Heinz, S. S. Hong, J. Huang, A. F. Ismach, E. Johnston-Halperin, M. Kuno, V. V. Plashnitsa, R. D. Robinson, R. S. Ruoff, S. Salahuddin, J. Shan, L. Shi, M. G. Spencer, M. Terrones, W. Windl and J. E. Goldberger, ACS Nano, 2013, 7, 28982926.

12 Y. Han, Y. Ge, Y. Chao, C. Wang and G. G. Wallace, J. Energy Chem., 2018, 27, 57-72.

13 A. H. Khan, S. Ghosh, B. Pradhan, A. Dalui, L. K. Shrestha, S. Acharya and K. Ariga, Bull. Chem. Soc. Jpn., 2017, 90, 627-648.

14 B. Anasori, M. R. Lukatskaya and Y. Gogotsi, Nat. Rev. Mater., 2017, 2, 16098.

15 A. H. Castro Neto, F. Guinea, N. M. R. Peres, K. S. Novoselov and A. K. Geim, Rev. Mod. Phys., 2009, 81, 109-162.

16 P. Vogt, P. De Padova, C. Quaresima, J. Avila, E. Frantzeskakis, M. C. Asensio, A. Resta, B. Ealet and G. Le Lay, Phys. Rev. Lett., 2012, 108, 155501.
17 P. De Padova, C. Quaresima, C. Ottaviani, P. M. Sheverdyaeva, P. Moras, C. Carbone, D. Topwal, B. Olivieri, A. Kara, H. Oughaddou, B. Aufray and G. Le Lay, Appl. Phys. Lett., 2010, 96, 261905.

18 K. Takeda and K. Shiraishi, Phys. Rev. B, 1994, 50, 1491614922.

19 G. G. Guzmán-Verri and L. C. Lew Yan Voon, Phys. Rev. B, 2007, 76, 075131.

20 S. Cahangirov, M. Topsakal, E. Aktürk, H. Şahin and S. Ciraci, Phys. Rev. Lett., 2009, 102, 236804.

21 C.-L. Lin, R. Arafune, K. Kawahara, M. Kanno, N. Tsukahara, E. Minamitani, Y. Kim, M. Kawai and N. Takagi, Phys. Rev. Lett., 2013, 110, 076801.

22 M. Rachid Tchalala, H. Enriquez, A. J. Mayne, A. Kara, S. Roth, M. G. Silly, A. Bendounan, F. Sirotti, T. Greber, B. Aufray, G. Dujardin, M. Ait Ali and H. Oughaddou, Appl. Phys. Lett., 2013, 102, 083107.

23 W. Zhang, L. Sun, J. M. V. Nsanzimana and X. Wang, Adv. Mater., 2018, 30(15), 1705523.

24 P. De Padova, H. Feng, J. Zhuang, Z. Li, A. Generosi, B. Paci, C. Ottaviani, C. Quaresima, B. Olivieri, M. Krawiec and Y. Du, J. Phys. Chem. C, 2017, 121, 27182-27190.

25 A. Fleurence, R. Friedlein, T. Ozaki, H. Kawai, Y. Wang and Y. Yamada-Takamura, Phys. Rev. Lett., 2012, 108, 245501.

26 T. H. Osborn and A. A. Farajian, J. Phys. Chem. C, 2012, 116, 22916-22920.

27 R. Qin, C.-H. Wang, W. Zhu and Y. Zhang, AIP Adv., 2012, 2, 022159.

28 T. Botari, E. Perim, P. a. S. Autreto, A. C. T. van Duin, R. Paupitz and D. S. Galvao, Phys. Chem. Chem. Phys., 2014, 16, 19417-19423.

29 X. Yuan, G. Lin and Y. Wang, Mol. Simul., 2016, 42, 11571164.

30 T. Rakib, S. Mojumder, S. Das, S. Saha and M. Motalab, Phys. $B, 2017, \mathbf{5 1 5}, 67-74$.

31 B. Feng, Z. Ding, S. Meng, Y. Yao, X. He, P. Cheng, L. Chen and $\mathrm{K} . \mathrm{Wu}$, Nano Lett., 2012, 12, 3507-3511.

32 H. Jamgotchian, B. Ealet, Y. Colignon, H. Maradj, J.-Y. Hoarau, J.-P. Biberian and B. Aufray, J. Phys.: Condens. Matter, 2015, 27, 395002.

33 N. Ding, H. Wang, X. Chen and C.-M. L. Wu, RSC Adv., 2017, 7, 10306-10315.

34 S. M. Nahid, F. H. Bhuiyan, T. Rakib and S. Mojumder, Effects of Defects on the Fracture Strength of Hexagonal Boron Nitride, unpublished.

35 T. Zhang, X. Li and H. Gao, Int. J. Fract., 2015, 196, 1-31.

36 T. Rakib, S. Saha, M. Motalab, S. Mojumder and M. M. Islam, Sci. Rep., 2017, 7, 14629.

37 T. Zhang, X. Li and H. Gao, J. Mech. Phys. Solids, 2014, 67, 213.

38 T. Zhang, X. Li and H. Gao, Extreme Mech. Lett., 2014, 1, 3-8. 39 F. Meng, C. Chen and J. Song, J. Phys. Chem. Lett., 2015, 6, 4038-4042.

40 G. Rajasekaran and A. Parashar, Diamond \& Related Materials, 2017, Complete, pp. 90-99.

41 E. Sandoz-Rosado, T. D. Beaudet, R. Balu and E. D. Wetzel, Nanoscale, 2016, 8, 10947-10955. 
42 X. Yang and J. Ni, Phys. Rev. B, 2005, 72, 195426.

43 J.-Y. Chung, V. Sorkin, Q.-X. Pei, C.-H. Chiu and Y.-W. Zhang, J. Phys. D: Appl. Phys., 2017, 50, 345302.

44 S. X. Gong and S. A. Meguid, Int. J. Mech. Sci., 1992, 34, 933945.

45 K. X. Hu, A. Chandra and Y. Huang, Int. J. Solids Struct., 1993, 30, 1473-1489.

46 T. Liu and S. Groh, Mater. Sci. Eng., 2014, 609, 255-265.

47 M. A. N. Dewapriya and S. A. Meguid, Carbon, 2017, 125, 113131.

48 M. A. N. Dewapriya and S. A. Meguid, Comput. Mater. Sci., 2018, 141, 114-121.

49 P. R. Budarapu, B. Javvaji, V. K. Sutrakar, D. Roy Mahapatra, G. Zi and T. Rabczuk, J. Appl. Phys., 2015, 118, 064307.

50 R. Abadi, R. P. Uma, M. Izadifar and T. Rabczuk, Comput. Mater. Sci., 2017, 131, 86-99.

51 H. Bao, Y. Huang, Z. Yang, Y. Sun, Y. Bai, Y. Miao, P. K. Chu, K. Xu and F. Ma, J. Phys. Chem. C, 2018, 122, 1351-1360.

52 A. Mattoni, L. Colombo and F. Cleri, Phys. Rev. Lett., 2005, 95, 115501.

53 C. Fabrizio, R. Phillpot Simon, W. Dieter and Y. Sidney, J. Am. Ceram. Soc., 2005, 81, 501-516.

54 S. Rouhi, Comput. Mater. Sci., 2017, 131, 275-285.

55 R. E. Roman and S. W. Cranford, Comput. Mater. Sci., 2014, 82, 50-55.

56 A. İnce and Ş. Erkoç, Phys. Status Solidi B, 2012, 249, 74-81.

57 Y. Jing, Y. Sun, H. Niu and J. Shen, Phys. Status Solidi B, 2013, 250, 1505-1509.

58 R. Ansari, S. Rouhi and S. Ajori, Superlattices Microstruct., 2014, 65, 64-70.

59 Q.-X. Pei, Z.-D. Sha, Y.-Y. Zhang and Y.-W. Zhang, J. Appl. Phys., 2014, 115, 023519.

60 M. R. Chávez-Castillo, M. A. Rodríguez-Meza and L. MezaMontes, RSC Adv., 2015, 5, 96052-96061.
61 M.-Q. Le and D.-T. Nguyen, Appl. Phys. A, 2015, 118, 14371445.

62 S. Plimpton, J. Comput. Phys., 1995, 117, 1-19.

63 M. A. N. Dewapriya, A. S. Phani and R. K. N. D. Rajapakse, Modell. Simul. Mater. Sci. Eng., 2013, 21, 065017.

64 T. H. Pial, T. Rakib, S. Mojumder, M. Motalab and M. A. S. Akanda, Phys. Chem. Chem. Phys., 2018, 20, 86478657.

65 D. H. Tsai, J. Chem. Phys., 1979, 70, 1375-1382.

66 F. H. Stillinger and T. A. Weber, Phys. Rev. B, 1985, 31, 52625271.

67 X. Zhang, H. Xie, M. Hu, H. Bao, S. Yue, G. Qin and G. Su, Phys. Rev. B, 2014, 89, 054310.

68 Q. Peng, X. Wen and S. De, RSC Adv., 2013, 3, 13772-13781.

69 T. L. Anderson, Fracture Mechanics: Fundamentals and Applications, CRC Press, 4th edn, 2017.

70 G. Pluvinage and L. Milovic, Fracture at all Scales, Springer International Publishing, 2017.

71 S. S. Terdalkar, S. Huang, H. Yuan, J. J. Rencis, T. Zhu and S. Zhang, Chem. Phys. Lett., 2010, 494, 218-222.

72 S. Huang, S. Zhang, T. Belytschko, S. S. Terdalkar and T. Zhu, J. Mech. Phys. Solids, 2009, 57, 840-850.

73 F. Liu, P. Ming and J. Li, Phys. Rev. B, 2007, 76, 064120.

74 M. A. N. Dewapriya, R. Rajapakse and A. S. Phani, in ICF13, 2013.

75 V. B. Shenoy, C. D. Reddy, A. Ramasubramaniam and Y. W. Zhang, Phys. Rev. Lett., 2008, 101, 245501.

76 Q. Lu and R. Huang, Phys. Rev. B, 2010, 81, 155410.

77 J. da Silva-Araújo, A. J. M. Nascimento, H. Chacham and R. W. Nunes, Nanotechnology, 2013, 24, 035708.

78 M. Buongiorno Nardelli, B. I. Yakobson and J. Bernholc, Phys. Rev. B, 1998, 57, R4277-R4280. 